\title{
sciendo
}

\author{
Current Issues in Pharmacy and Medical Sciences
}

Formerly ANNALES UNIVERSITATIS MARIAE CURIE-SKIODOWSKA, SECTIO DDD, PHARMACIA

journal homepage: http://www.curipms.umlub.pl/

\section{Molecular docking study of the acetylcholinesterase inhibition}

\author{
Amina Merzoug ${ }^{1,2 \star}\left({ }^{\circ}\right.$, Hanane Boucherit $^{1,2}$, Rima KhaleD $^{2}$, \\ Amel Chefiri $^{2}$, Abdelouahab Chikhi $^{1}$, Abderrahmane Bensegueni ${ }^{1}$
}

\begin{abstract}
${ }^{1}$ Laboratory of Applied Biochemistry, Department of Biochemistry and Cellular and Molecular Biology, Faculty of Natural and Life Sciences, Mentouri Brothers University, Algeria

${ }^{2}$ Abdelhafid Boussouf University Center, Mila, Algeria
\end{abstract}

\section{ARTICLE INFO \\ Received 12 November 2020 \\ Accepted 02 January 2021}

\section{Keywords:}

acetylcholinesterase,

Alzheimer's disease,

FlexX,

molecular docking,

ZINC database.

\begin{abstract}
While Alzheimer disease is the most common form of dementia, acetylcholinesterase is an interesting therapeutic target for the development of new anti-Alzheimer's disease drugs. In order to discover potential compounds inhibiting this protein target, a molecular docking study of a similar collection of 1-[[2,4-bis[(E)hydroxyiminomethyl] pyridin-1ium-1-yl]methoxymethyl] pyridin-1-ium-4-carboxamide (HLO) inhibitor from ZINC database using FlexX program was realized. Before performing the molecular docking, FlexX was validated by Root mean square deviation test to determine the reproducibility of the docking program. The strategy undertaken in this study permitted us to propose products 4-[[2-[(Z)-N'-hydroxycarbamimidoyl]-4-pyridyl]methylamino] benzamide and $\mathrm{N}$-[(E)-[1-(4-nitrophenyl)pyrrol-2-yl]methylene amino]isonicotinamide as potential new inhibitors of humane acetylcholinesterase. The two proposed products may act as strong anti-Alzheimer leads compounds.
\end{abstract}

\section{INTRODUCTION}

Alzheimer's disease is a chronic neurodegenerative pathology, accounting for $60-70 \%$ of all cases of dementia and touching about $6 \%$ of all persons 65 years and older $[1,2]$. Many studies relate the deteriorated cognitive functions of Alzeheimer patients with the reduced synthesis of the neurotransmitter acetylcholine that is released into the synapses of the nervous system [3-5]. Acetylcholinesterase (AChE; EC 3.1.1.7) is a critical enzyme that regulates neurotransmission by catalyzing the degradation of the acetylcholine [6]. The capacity of this enzyme increases in Alzheimer's patients, which make it a target of interest in the search of new treatment [7].

Four anti-AChE agents are currently used as Alzheimer's disease drugs. These are tacrine, rivastigmine, donepezil and galantamine [8]. However, the application of these drugs is limited by their severe side effects, including hepatotoxic liability [9], muscle cramps [10] diarrhea and vomiting [11,12]. Therefore, it is still necessary to develop new products to treat Alzheimer's disease. Structural studies of the AChE active site show that it has two adjacent pockets to which ligands may bind - the peripheral pocket at the entrance of the cavity and the catalytic pocket at its bottom [13-16].

\footnotetext{
* Corresponding author

e-mail: a.merzouk@centre-univ-mila.dz
}

In the catalytic pocket, the Ser203, His447 and Glu334 are the key residues for the substrate hydrolyzation [13]. Tyr72, Tyr124 and Trp286 amino acids play an essential role in the interaction between the ligand and the peripheral pocket.

The object of the present work was to analyze the interactions involved in the inhibition of $\mathrm{AChE}$ by various compounds listed in the Protein Data Bank (PDB) and in a collection of derivatives of the inhibitor that had the greatest affinity, by means of applying the molecular docking method. This is one of the commonly used computational strategies in structure-based drug design [17]. The intent is to realize a computational absorption, distribution, metabolism, excretion and toxicity (ADME/Tox) prediction for exploring the best possible inhibitors for AChE. These actions may aid the development of more efficient anti-Alzheimer's disease agents.

\section{MATERIALS AND METHOD}

\section{Protein preparation}

The enzyme selected as a pharmacological target for the work was acetylcholinesterase, the 3D structure of which was retrieved from the PDB (PDB ID: 4M0E) [18]. It has two identical chains (A, B). In the protein structure preparation, chain $B$ was removed and the co-crystal selective inhibitor 1YL was used for active site determination. The 
residues Tyr72, Asp74, Gly121, Tyr124, Trp286, His287, Leu289, Gln291, Glu292, Ser293, Val294, Phe295, Arg296, Phe297, Tyr337, Phe338, Tyr341, Gly342 His447 and four water molecules were found to be present in the binding pocket of the proposed target enzyme.

\section{Molecular docking}

In order to assess by means of the molecular docking approach, the interaction modes and orientation on the $\mathrm{AChE}$ binding pocket of 82 inhibitors listed in the PDB and in a collection of 119 derivatives of the inhibitors that had the greatest affinity to the binding site, we used the most recent version of FlexX software $(2.3 .3,2017)$.

The three-dimensional structures of the derivative compounds were downloaded in the sdf. format from the ZINC database (http://zinc.docking.org), a commercially available library of 35 million chemical products with vendors and product proprieties information.

\section{ADME/Tox}

To predict the pharmacokinetic properties of the most promising products presaged by molecular docking, a computational ADME/Tox study was performed using Suissadme (http://www.swissadme.ch) for blood-brain barrier (BBB) penetration, gastrointestinal absorption (GI), and Cytochrome P450 (CYP) inhibition. Moreover, PreADMET (https://preadmet.bmdrc.kr) was employed to establish cell permeability (COCA-2) and toxicity.

All potential drugs had to met Lipinski's Rule of Five, which states that likely orally active drug must complete two of these four proprieties [19]:

- no more than 5 hydrogen bond donors;

- no more than 10 hydrogen bond acceptors;

- the molecular weight under $500 \mathrm{~g} / \mathrm{mol}^{-1}$;

- the Log P below 5 .

\section{RESULTS AND DISCUSSION}

\section{Ability of docking protocol}

Before performing the molecular docking study, the docking protocol was validated. The co-crystal selective ligands were, therefore, removed from 240 crystallized protein-ligand complexes listed in the PDB (Tab. 1) and again docked back into the binding site of the proteins. Root mean square deviation (RMSD) of $78.55 \%$ of the ligands in co-crystal complex conformations and the best-docked conformations were less than $2 \AA$. Most ligands showed deviations that were negligible (Fig. 1). This indicated the capacity of the docking protocol to reproduce the interaction modes and orientations of the co-crystal ligands [20].

Study of the interactions involved in the inhibition of AChE by various compounds

With the aim of identifying new inhibitors of AChE, we studied firstly the mechanism of inhibition set up by 82 known inhibitors itemized in the PDB. The results of the simulation by FlexX of these inhibitors are represented below in Table 2 .

The results presented in Table 2 reveal that among the 82 docked inhibitors, compound 1-[[2,4-bis[(E) hydroxyiminomethyl] pyridin-1-ium-1-yl]methoxymethyl]

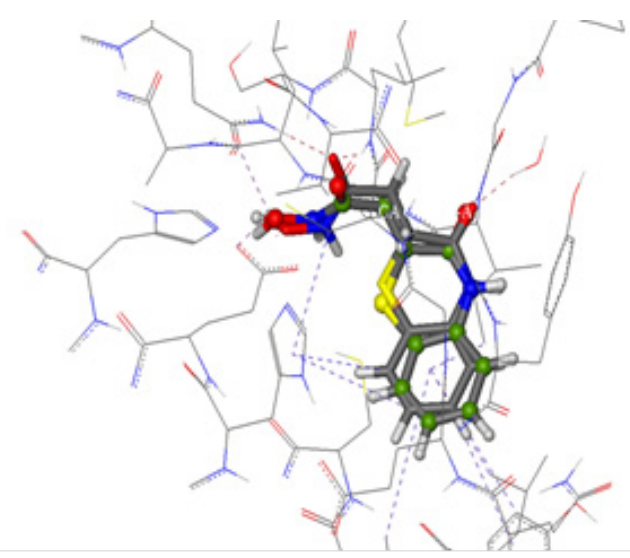

Figure 1. Overlay of co-crystal ligand NLP conformation extracted from $2 \mathrm{GU} 4$ (colored in grey) with the best-docked conformation (colored in green)

pyridin-1-ium-4-carboxamide (HLO) demonstrated the highest score, and hence, is the most potent inhibitor of AchE. For this reason, we have chosen this compound as reference model to interpret the diverse established links with the protein in question, and as starting structure for the development of new potent inhibitors.

The presented docking study reveals that the compound $\mathrm{HLO}$ fits favorably into the AChE binding pocket as it displays hydrogen bonds with Trp286, Leu289, Ser293, Tyr337 (Fig. 2), as well as hydrophobic interactions with Tyr72, Tyr124, Trp286, His287, Leu289, Tyr341 (Fig. 3) with a binding energy of $-34,4450 \mathrm{kj} / \mathrm{mol}$.

Accordingly:

- A hydrogen bridge is observed between the carbonyl of the inhibitor and the water molecule $\mathrm{H} 2 \mathrm{O} 901$ present in the binding pocket $(\mathrm{O} 1 \ldots \ldots . . \mathrm{H}-\mathrm{H} 2 \mathrm{O} 901)$;

- Two hydrogen bridges are observed between the amine function of the inhibitor and the two carbonyl groups of Trp286 (N3-H......... O-Trp286) and Leu289 (N3-H..........-Leu289);

- Two hydrogen bonds are observed between the nitrogen atoms of the inhibitor and the water molecules $\mathrm{H} 2 \mathrm{O} 715$ $(\mathrm{N} 16 \ldots \ldots \ldots . \mathrm{H}-\mathrm{H} 2 \mathrm{O} 715)$ and $\mathrm{H} 2 \mathrm{O} 857$ (N23 ........ $\mathrm{H}-\mathrm{H} 2 \mathrm{O} 857$ ) presents in the binding site;

- Two hydrogen bridges are established between the hydroxyl group of the inhibitor and the carbonyl function of Ser293 (H-O17..........-Ser293) on the one hand and the hydroxyl function of Tyr337 on the other hand (H-O14......... O-Tyr337).

The ZINC database was used to find the structural analogues of the inhibitor HLO in order to dock them by FlexX on the human $\mathrm{AChE}$ crystallographic structure, $4 \mathrm{M} 0 \mathrm{E}$, and to search among them for those that bind more strongly than the HLO to our target AChE. We then compared the binding energies of these compounds (Tab. 3) with the reference inhibitor, HLO, and proposed the best inhibitors of the enzyme in question.

Of the 119 inhibitors tested, the compounds 4-[[2-[(Z)N'-hydroxycarbamimidoyl]-4-pyridyl]methylamino] benzamide (ZINC78954474) and N-[(E)-[1-(4-nitrophenyl)pyrrol2-yl]methylene amino]isonicotinamide (ZINC00123616) showed lower binding energies than the reference inhibitor (Tab. 3). They also formed the most stable AChE-inhibitor 
complexes. Hence, we propose that these two compounds then undertaken to elucidate the mechanism of interaction can interact efficiently with the enzyme. Further work was

of these proposed compounds.

Table 1. List of 240 complexes used to test the ability of the docking protocol

\begin{tabular}{|c|c|c|c|c|c|c|c|c|}
\hline Enzyme code & Inhibitor code & $\operatorname{RMSD}(\AA)$ & Enzyme code & Inhibitor code & $\operatorname{RMSD}(\AA)$ & Enzyme code & Inhibitor code & $\operatorname{RMSD}(\AA)$ \\
\hline $4 \mathrm{MOE}$ & $1 \mathrm{YL}$ & 0.9502 & $2 \mathrm{HA} 3$ & CHT & 2.5355 & $2 X \cup Q$ & TZ4 & 1.3917 \\
\hline $5 F O G$ & GCB & 0.7738 & $2 J 3 Q$ & TFL & 0.6751 & 3DL4 & P6G & 5.9637 \\
\hline $1 \mathrm{AX} 9$ & EDR & 0.5188 & 2JEY & HLO & 1.0675 & $3 Z L T$ & PGO & 2.9450 \\
\hline $2 \mathrm{ACK}$ & EDR & 0.3768 & $2 \mathrm{JFO}$ & HBP & 2.0238 & $3 Z L V$ & HI6 & 0.3776 \\
\hline $2 \mathrm{WHR}$ & K27 & 0.3881 & 2VQ6 & FP1 & 1.2423 & $4 A 23$ & C56 & 0.4103 \\
\hline 3M3D & PG4 & 1.3365 & $2 W L S$ & $\mathrm{X} 13$ & 0.8930 & 4ARA & C56 & 1.0888 \\
\hline $1 \mathrm{DX} 6$ & GNT & 0.5148 & $6 \mathrm{CQW}$ & HI6 & 0.8212 & 4ARB & C57 & 0.7511 \\
\hline $1 \mathrm{E} 66$ & HUX & 0.5944 & $6 \mathrm{CQU}$ & HI6 & 4.3540 & $4 B 7 Z$ & PEG & 1.5925 \\
\hline $1 \mathrm{GPN}$ & HUB & 0.6544 & $6 \mathrm{CQV}$ & HI6 & 2.8438 & $4 \mathrm{~B} 8 \mathrm{O}$ & PEG & 1.7046 \\
\hline $2 \mathrm{C} 58$ & ETM & 0.6814 & $6 \mathrm{EZH}$ & $\mathrm{C} 6 \mathrm{H}$ & 1.7114 & 4B81 & PEG & 2.0632 \\
\hline $2 \mathrm{JEZ}$ & HLO & 0.7767 & $6 \mathrm{EZG}$ & $\mathrm{C} 6 \mathrm{H}$ & 2.5989 & 4B82 & B3Z & 1.6736 \\
\hline $3 Z L U$ & $1 \mathrm{KA}$ & 1.5215 & $50 \mathrm{~V} 9$ & PE4 & 2.7692 & 4B83 & B3V & 1.1221 \\
\hline 6EWK & RMO & 0.5662 & $1 \mathrm{~N} 5 \mathrm{M}$ & GMN & 1.5301 & 4B84 & Z5K & 2.0024 \\
\hline 2XUD & DME & 1.3136 & $1 \mathrm{J07}$ & $\mathrm{DCU}$ & 2.2569 & 4B85 & B3W & 1.4123 \\
\hline $1 \mathrm{ACL}$ & DME & 1.7168 & 1306 & AE3 & 2.9054 & $4 \mathrm{BCO}$ & SO4 & 0.7938 \\
\hline $1 \mathrm{ACJ}$ & THA & 0.5243 & 3DL7 & P6G & 0.9210 & 4BDT & HUW & 0.5076 \\
\hline $5 \mathrm{E} 4 \mathrm{~J}$ & DME & 1.5373 & 2JGK & P6G & 2.6636 & $4 \mathrm{EY} 5$ & HUP & 0.3100 \\
\hline 5DLP & SO4 & 0.9518 & $2 \mathrm{JGJ}$ & AE3 & 2.8217 & 5HF5 & EDO & 0.5691 \\
\hline 5EIE & ACT & 0.4865 & $2 \mathrm{JGF}$ & P6G & 4.1626 & $5 \mathrm{HF6}$ & EDO & 0.5898 \\
\hline $1 \mathrm{Q} 84$ & TZ4 & 0.5954 & $2 J G L$ & P6G & 2.7171 & 5HF8 & EDO & 0.8023 \\
\hline $3 \mathrm{ZV7}$ & PEG & 0.6458 & $2 \mathrm{JGI}$ & AE3 & 5.0399 & 5HF9 & HI6 & 1.3671 \\
\hline $1 \mathrm{~N} 5 \mathrm{R}$ & $1 \mathrm{CY}$ & 0.8627 & $6 \mathrm{CQZ}$ & vx & 1.5635 & 5HFA & FP1 & 0.7177 \\
\hline $2 \times 8 B$ & SO4 & 0.6766 & $6 \mathrm{CQX}$ & $7 P E$ & 2.5366 & $4 \mathrm{EY} 7$ & GNT & 0.3916 \\
\hline $5 \mathrm{EIH}$ & ACT & 0.4289 & $5 \mathrm{HQ} 3$ & MES & 1.5038 & $4 \mathrm{EY} 8$ & E20 & 0.7238 \\
\hline $5 \mathrm{EHQ}$ & 502 & 1.0668 & 5E2I & PEG & 1.4563 & 4TVK & SO4 & 0.3913 \\
\hline $1 \mathrm{GQS}$ & SAF & 0.6484 & 5EIE & PG4 & 2.6792 & $4 W 63$ & $\mathrm{TJH}$ & 1.0833 \\
\hline 1VOT & HUP & 0.4708 & 5EIA & PG4 & 4.7369 & $4 \times 3 C$ & HIB & 3.9986 \\
\hline $2 \mathrm{C} 4 \mathrm{H}$ & PGE & 1.4454 & $5 \mathrm{EHZ}$ & $5 \mathrm{NZ}$ & 1.6242 & 5BWB & TNH & 3.6490 \\
\hline $2 \mathrm{C} 5 \mathrm{G}$ & ETM & 0.6527 & $5 \mathrm{EHN}$ & P6G & 3.0123 & $5 \mathrm{BWC}$ & $4 \mathrm{VV}$ & 1.8078 \\
\hline $2 \mathrm{HAO}$ & P6G & 1.5995 & $2 \mathrm{HA} 2$ & SCK & 1.1327 & 5DTJ & HBP & 0.9884 \\
\hline $2 \times \cup 0$ & TZ4 & 2.5338 & $2 \mathrm{HA5}$ & ACT & 0.3527 & $5 \mathrm{E} 4 \mathrm{~T}$ & $5 \mathrm{~GB}$ & 2.4733 \\
\hline 2XUP & TZ5 & 1.2976 & 2XI4 & AFT & 0.5179 & 5EHX & MBT & 0.7210 \\
\hline $2 X \cup Q$ & TZ4 & 1.3917 & $5 \mathrm{FPP}$ & HI6 & 0.6425 & 5EI5 & AE4 & 3.1581 \\
\hline $4 \mathrm{EY} 6$ & AA7 & 3.0474 & $1 \mathrm{C} 24$ & PFU & 6.6307 & $3 \pi T 2$ & O7N & 0.5873 \\
\hline $4 \mathrm{U} 73$ & Q02 & 0.4094 & $2 \mathrm{GG} 2$ & M12 & 1.1639 & 4EM7 & ORA & 0.7011 \\
\hline $5 L Y X$ & $7 \mathrm{BF}$ & 1.9080 & $2 \mathrm{GG} 3$ & M13 & 1.1867 & 4EMV & OR9 & 0.4311 \\
\hline 5IB9 & BES & 0.6464 & 2GGB & U17 & 0.9611 & 4LPO & $1 Y M$ & 0.6500 \\
\hline $4 X N D$ & HT7 & 0.7431 & $2 O A Z$ & 196 & 1.3077 & $4 \mathrm{LPB}$ & $1 \mathrm{YP}$ & 0.6344 \\
\hline $3 Q 7 J$ & FBO & 1.6734 & 2P99 & $2 \mathrm{P} 9 \mathrm{~A}$ & 0.4150 & $5 \mathrm{BOC}$ & TSJ & 2.4491 \\
\hline $1 \mathrm{G} 27$ & BB1 & 0.9022 & 3 IU7 & $1 \mathrm{XNZ}$ & 0.5235 & 5BS8 & MFX & 3.6761 \\
\hline 4EOX & OS5 & 1.1344 & 2ADU & 2ADU & 0.5179 & 5BTA & MFX & 1.2867 \\
\hline $2 \mathrm{AI} 8$ & SB7 & 0.9193 & 2BB7 & QMS & 0.1594 & 5BTC & $\mathrm{CPF}$ & 1.5176 \\
\hline $3 \mathrm{M} 6 \mathrm{Q}$ & BB2 & 0.9429 & $2 \mathrm{GU} 4$ & NLP & 0.0283 & 5BTD & GNF & 4.1934 \\
\hline $3 M 6 R$ & BB2 & 1.0015 & $2 \mathrm{GU} 5$ & NLP & 1.1581 & 5BTF & GNF & 7.7439 \\
\hline $303 \mathrm{~J}$ & BB4 & 0.6533 & $2 \mathrm{GU} 6$ & NLP & 0.6433 & 5BTG & LFX & 3.3794 \\
\hline $3 \mathrm{U} 7 \mathrm{~K}$ & MDB & 0.6623 & $2 \mathrm{Q} 92$ & B23 & 0.6552 & 5BTI & LFX & 4.8256 \\
\hline $3 U 7 M$ & $\mathrm{FHF}$ & 4.0437 & $2 \mathrm{Q} 93$ & B21 & 0.4706 & 5BTL & $8 M X$ & 3.2485 \\
\hline $3 U 7 N$ & UHF & 2.0083 & $2 Q 94$ & A04 & 0.4430 & $3 Q \times 3$ & EVP & 5.1885 \\
\hline $5 \mathrm{CWY}$ & $4 \mathrm{WL}$ & 0.4652 & $2 Q 95$ & A05 & 0.7386 & $4 \mathrm{GO} 4$ & ASN & 1.5770 \\
\hline
\end{tabular}


Amina Merzoug, Hanane Boucherit, Rima Khaled, Amel Chefiri, Abdelouahab Chikhi, Abderrahmane Bensegueni

\begin{tabular}{|c|c|c|c|c|c|c|c|c|}
\hline $5 \mathrm{CY} 7$ & $56 U$ & 0.6061 & $2 \mathrm{Q} 96$ & $\mathrm{~A} 18$ & 0.5771 & $4 \mathrm{GOV}$ & MIX & 6.1906 \\
\hline $5 \mathrm{CY} 8$ & $56 \mathrm{~V}$ & 0.4530 & 4IF7 & $\mathrm{HCM}$ & 0.7846 & $465 \mathrm{D}$ & $9 A D$ & 0.7673 \\
\hline 5MTE & BB2 & 0.8852 & $4 \mathrm{Z7M}$ & $427 \mathrm{M}$ & 1.5471 & $1 \mathrm{CYO}$ & $\mathrm{A} 3 \mathrm{P}$ & 5.9194 \\
\hline $5 \mathrm{JF} 1$ & BB2 & 0.9775 & $1 \mathrm{C} 27$ & NLP & 0.8954 & $1 \mathrm{CY} 7$ & $\mathrm{PO} 4$ & 1.2626 \\
\hline $5 \mathrm{JF} 6$ & BB4 & 0.7885 & $2 \mathrm{EVC}$ & $\mathrm{FC} 3$ & 0.5437 & $3 \mathrm{FOC}$ & NFX & 4.8770 \\
\hline 5JF8 & PN3 & 0.5193 & $2 \mathrm{G} 6 \mathrm{P}$ & HM2 & 1.0061 & $3 \mathrm{FV} 5$ & $1 \mathrm{EU}$ & 0.5342 \\
\hline 3PN3 & BN3 & 3.1723 & 4FLL & YZ6 & 1.1956 & $4 Z 4 Q$ & PDQ & 2.7545 \\
\hline 3PN4 & $\mathrm{BD} 2$ & 0.8548 & EVO & Сто & 1.4476 & 1L8I & EHD & 3.5061 \\
\hline 2AIA & SB8 & 1.5430 & 2EVM & $\mathrm{FC2}$ & 0.3260 & 1T8I & $\pi \mathrm{J}$ & 3.5061 \\
\hline $5 \mathrm{VCP}$ & BB2 & 0.8185 & 3PKB & Y16 & 0.7731 & 5BOD & RXV & 0.9883 \\
\hline $1 \mathrm{G} 2 \mathrm{~A}$ & BB2 & 0.7577 & $3 \mathrm{PKC}$ & Y08 & 1.1691 & $2 \times C S$ & $\mathrm{RXV}$ & 3.9899 \\
\hline $1 \mathrm{~S} 17$ & GNR & 0.3008 & 4FLJ & Y08 & 1.1691 & 5L 3J & $6 \mathrm{G9}$ & 0.9875 \\
\hline 1WS1 & BB2 & 0.9348 & 1R5G & A01 & 1.4255 & $2 \times C T$ & CPF & 2.9560 \\
\hline 2EW5 & $\mathrm{Y} 12$ & 1.3292 & 1R5H & A02 & 0.9170 & $4 \mathrm{~K} 40$ & DOO & 1.2477 \\
\hline 2EW6 & Y13 & 0.9684 & 4CPM & G39 & 0.4185 & 3CL0 & G39 & 0.3994 \\
\hline 2KMN & BB2 & 1.4302 & 4GZP & G39 & 0.6194 & $2 \mathrm{HT7}$ & G39 & 0.5128 \\
\hline 1LQY & BB2 & 0.6687 & $4 \mathrm{HZZ}$ & G39 & 0.3319 & 6EKU & ZMR & 0.6360 \\
\hline 1LRU & BB2 & 0.8999 & $5 B 2 D$ & SLT & 3.2417 & 1IVD & ST1 & 0.8785 \\
\hline $3 \mathrm{G} 5 \mathrm{~K}$ & BB2 & 0.7379 & $5 \mathrm{KKY}$ & $6 \mathrm{VD}$ & 0.6277 & 3K37 & $\mathrm{BCZ}$ & 0.3368 \\
\hline 3K6I & $2 \mathrm{BB}$ & 0.4604 & $1 \mathrm{~A} 4 \mathrm{Q}$ & DPC & 0.8848 & $4 \mathrm{HZW}$ & LNV & 0.4672 \\
\hline $3 \mathrm{M} 6 \mathrm{P}$ & BB2 & 0.8042 & 1B9T & RAI & 0.8268 & $4 \mathrm{HZX}$ & G39 & 0.3692 \\
\hline 4DR9 & BB2 & 1.0375 & $1 \mathrm{~B} 9 \mathrm{~V}$ & RA2 & 0.6461 & $4 \mathrm{MJU}$ & 275 & 0.7077 \\
\hline $4 \mathrm{U} 73$ & $\mathrm{Q} 02$ & 0.4094 & $2 Q W D$ & $4 \mathrm{AM}$ & 0.5061 & $4 \mathrm{MJV}$ & $27 \mathrm{~V}$ & 1.2499 \\
\hline $117 \mathrm{H}$ & $\mathrm{BCZ}$ & 0.5295 & 1 LRY & BB2 & 0.9257 & 4QN6 & LNV & 0.4378 \\
\hline $3 \mathrm{H} 73$ & DNA & 0.5718 & $1 \mathrm{Q} 1 \mathrm{Y}$ & BB2 & 2.3749 & $5 \mathrm{KV9}$ & I57 & 1.1471 \\
\hline $3 T 13$ & LNV & 0.4328 & $1 \mathrm{SSZ}$ & BB2 & 1.2646 & 1INH & ST6 & 0.8687 \\
\hline $3 T 16$ & G39 & 0.3460 & 2053 & BB2 & 0.8713 & $1 \mathrm{IFF}$ & $\mathrm{BCZ}$ & 0.3540 \\
\hline $3 T 18$ & LNV & 0.4535 & $3 E 3 U$ & NVC & 0.7303 & $1 \mathrm{IIG}$ & $\mathrm{BCZ}$ & 0.4746 \\
\hline 4JE7 & BB2 & 0.8267 & 4E9A & QAP & 0.5152 & 5T8Z & BB2 & 0.7091 \\
\hline $512 B$ & BB2 & 0.7713 & 3CL0 & G39 & 0.3994 & $2 \mathrm{HT7}$ & G39 & 0.5128 \\
\hline
\end{tabular}

Table 2. The Interaction energy $(\mathrm{kj} / \mathrm{mol})$ of $\mathrm{AChE}$ and studied inhibitors obtained by molecular docking

\begin{tabular}{|c|c|c|c|c|c|c|c|}
\hline Ligand code & $\Delta \mathrm{G}(\mathrm{kj} / \mathrm{mol})$ & Ligand code & $\Delta \mathrm{G}(\mathrm{kj} / \mathrm{mol})$ & Ligand code & $\Delta \mathrm{G}(\mathrm{kj} / \mathrm{mol})$ & Ligand code & $\Delta \mathrm{G}(\mathrm{kj} / \mathrm{mol})$ \\
\hline $4 \mathrm{VV}$ & -15.8172 & GB & -12.0604 & B3W & -17.4707 & P6G & -10.9246 \\
\hline $5 G B$ & -13.6516 & GL8 & -13.8893 & B3V & -23.5786 & SCK & -12.2243 \\
\hline $5 G Z$ & -24.95 & GMN & -15.4150 & AA7 & -3.1715 & VXA & -12.0419 \\
\hline ZN4 & -20.7391 & GNT & -15.6246 & A36 & -23.1579 & SO4 & -11.6581 \\
\hline TZ5 & -10.5999 & HUX & -13.8401 & ATJ & -13.1162 & AE4 & -8.9338 \\
\hline TNH & -21.7430 & I40 & -18.5550 & ETM & -7.9629 & $1 \mathrm{PE}$ & -11.7707 \\
\hline $\mathrm{TJH}$ & -30.7445 & NAF & -16.1984 & HLO & -34.7418 & DEP & -14.0186 \\
\hline SOF & -24.0018 & NDG & -20.7377 & MF2 & -12.6524 & $v x$ & -15.0290 \\
\hline Q4Q & -21.5731 & NWA & -10.2082 & NTJ & -14.2055 & EFS & -12.8749 \\
\hline PE4 & -12.0153 & PRM & -9.1282 & OBI & -25.9574 & $5 G 8$ & -13.6516 \\
\hline P15 & -9.3975 & SAF & -19.0554 & PGE & -11.3268 & G6X & -10.9728 \\
\hline HUW & -16.1830 & SCU & -11.1420 & TFL & -23.8970 & G3X & -11.8505 \\
\hline HUP & -14.6125 & THA & -15.2270 & FP1 & -19.4458 & AFT & -22.8637 \\
\hline HTB & -7.1524 & $\mathrm{~K} 27$ & -22.1019 & GC8 & -19.7172 & FP1 & -17.4727 \\
\hline HI6 & -28.3230 & $\mathrm{ACH}$ & -11.9886 & NHG & -19.8947 & BMA & -14.4306 \\
\hline HBP & -20.0967 & AT3 & -9.8184 & $\mathrm{PZ5}$ & -15.0158 & $\mathrm{CHT}$ & -8.5180 \\
\hline EFS & -12.8749 & $\mathrm{X} 13$ & -1.4202 & Z5K & -13.9569 & DCU & -21.2847 \\
\hline E20 & -27.5948 & DME & -4.6489 & $\mathrm{~A} 1 \mathrm{E}$ & -6.4493 & E10 & -11.0574 \\
\hline C57 & -26.4061 & H34 & -11.8740 & $\mathrm{~A} 2 \mathrm{E}$ & -4.6047 & EDR & -15.4352 \\
\hline C56 & -31.7773 & $\mathrm{NO} 3$ & -9.5901 & $\mathrm{~A} 8 \mathrm{~B}$ & -11.7649 & FBQ & -20.3385 \\
\hline B3Z & -19.4197 & NAG & -18.5179 & $\mathrm{~A} 8 \mathrm{~N}$ & -11.8723 & FUL & -16.2992 \\
\hline
\end{tabular}


Molecular docking study of the acetylcholinesterase inhibition

Table 3. FlexX score of HLO derivatives in AChE active site

\begin{tabular}{|c|c|c|c|c|c|}
\hline ZINC ID & $\begin{array}{c}\Delta \mathrm{G} \\
(\mathrm{kj} / \mathrm{mol})\end{array}$ & ZINC ID & $\begin{array}{c}\Delta \mathrm{G} \\
(\mathrm{kj} / \mathrm{mol})\end{array}$ & ZINC ID & $\begin{array}{c}\Delta \mathrm{G} \\
(\mathrm{kj} / \mathrm{mol})\end{array}$ \\
\hline ZINC00005077 & -27.3784 & ZINC68758087 & -21.4063 & ZINC68758091 & -15.5140 \\
\hline ZINC05033416 & -22.2578 & ZINC04577910 & -16.5619 & ZINC92984347 & -17.4974 \\
\hline ZINC01863576 & -22.6564 & ZINC15007645 & -24.3008 & ZINC92984332 & -21.3727 \\
\hline ZINC19913394 & -27.1784 & ZINC18056228 & -20.7744 & ZINC78990672 & -28.0314 \\
\hline ZINC05018113 & -25.4189 & ZINC70603710 & -24.1592 & ZINC79040001 & -23.7272 \\
\hline ZINC01846393 & -26.4366 & ZINC33288079 & -31.6016 & ZINC68758496 & -22.4302 \\
\hline ZINC95115865 & -22.7513 & ZINC78990209 & -27.1988 & ZINC15007565 & -24.7233 \\
\hline ZINC05171075 & -22.5787 & ZINC78990204 & -28.0080 & ZINC70603431 & -25.3969 \\
\hline ZINC20293508 & -20.3434 & ZINC68758023 & -19.0376 & ZINC82668666 & -20.5966 \\
\hline ZINC20293511 & -19.7444 & ZINC68757301 & -18.1518 & ZINC70604507 & -24.1599 \\
\hline ZINC01895559 & -24.2650 & ZINC82668669 & -19.1049 & ZINC70605318 & -24.2189 \\
\hline ZINC00307025 & -26.4089 & ZINC82668692 & -22.4096 & ZINC78986989 & -24.7547 \\
\hline ZINC11681703 & -23.7689 & ZINC82668695 & -20.2353 & ZINC68759091 & -23.3560 \\
\hline ZINC01777522 & -24.6313 & ZINC00123616 & -35.4759 & ZINC78956342 & -22.1763 \\
\hline ZINC91363880 & -24.3836 & ZINC28861846 & -26.9394 & ZINC78988308 & -23.9199 \\
\hline ZINC00174403 & -29.0414 & ZINC90349179 & -21.6062 & ZINC87753414 & -22.5928 \\
\hline ZINC13126573 & -30.1319 & ZINC05421373 & -17.3247 & ZINC87028628 & -26.9838 \\
\hline ZINC78954474 & -35.4099 & ZINC68759026 & -24.3144 & ZINC78987388 & -31.1159 \\
\hline ZINC68757759 & -16.9849 & ZINC68758488 & -20.8566 & ZINC78995801 & -26.2334 \\
\hline ZINC68757566 & -18.9321 & ZINC68758483 & -22.7008 & ZINC78989001 & -29.5646 \\
\hline ZINC82668672 & -20.5304 & ZINC78948412 & -23.7081 & ZINC68756848 & -20.7484 \\
\hline ZINC00520350 & -20.3053 & ZINC78949582 & -29.5121 & ZINC79003564 & -25.4209 \\
\hline ZINC68757623 & -16.2203 & ZINC68758180 & -16.5002 & ZINC68758213 & -21.3615 \\
\hline ZINC68758073 & -16.5997 & ZINC01791555 & -21.9668 & ZINC68758208 & -16.3030 \\
\hline ZINC68757928 & -18.8490 & ZINC68757981 & -15.2485 & ZINC68757977 & -20.7600 \\
\hline ZINC70603412 & -25.1510 & ZINC78990041 & -25.7528 & ZINC68757973 & -21.9547 \\
\hline ZINC70605338 & -28.5457 & ZINC68757614 & -23.1438 & ZINC79041148 & -27.6648 \\
\hline ZINC70604548 & -26.6129 & ZINC78997231 & -23.3699 & ZINC68758131 & -17.1508 \\
\hline ZINC68757943 & -20.0104 & ZINC68758049 & -18.5937 & ZINC68758128 & -15.9025 \\
\hline ZINC68757766 & -21.9830 & ZINC95221258 & -22.3941 & ZINC79038379 & -30.3929 \\
\hline ZINC68758423 & -20.2543 & ZINC72226005 & -20.6572 & ZINC79038382 & -27.7927 \\
\hline ZINC68758424 & -21.2133 & ZINC84846983 & -24.3246 & ZINC79041146 & -30.2255 \\
\hline ZINC01851448 & -24.2399 & ZINC78988661 & -22.6683 & ZINC68756925 & -22.8423 \\
\hline ZINC00436480 & $-34,5971$ & ZINC78988670 & -24.6570 & ZINC68756887 & -22.4208 \\
\hline ZINC70605287 & -27.2475 & ZINC90283585 & -26.6404 & ZINC68756891 & -21.2897 \\
\hline ZINC70605288 & -29.0262 & ZINC90283584 & -29.0710 & ZINC68756895 & -21.4838 \\
\hline ZINC68757720 & -22.2259 & ZINC68757472 & -20.7773 & ZINC68756920 & -23.7749 \\
\hline ZINC79041832 & -25.5717 & ZINC05119145 & -24.2329 & ZINC79007147 & -25.1192 \\
\hline ZINC79041837 & -25.4007 & ZINC68758621 & -17.5570 & ZINC82668669 & -19.1049 \\
\hline ZINC68758082 & -21.1319 & ZINC68758076 & -17.2070 & & \\
\hline
\end{tabular}




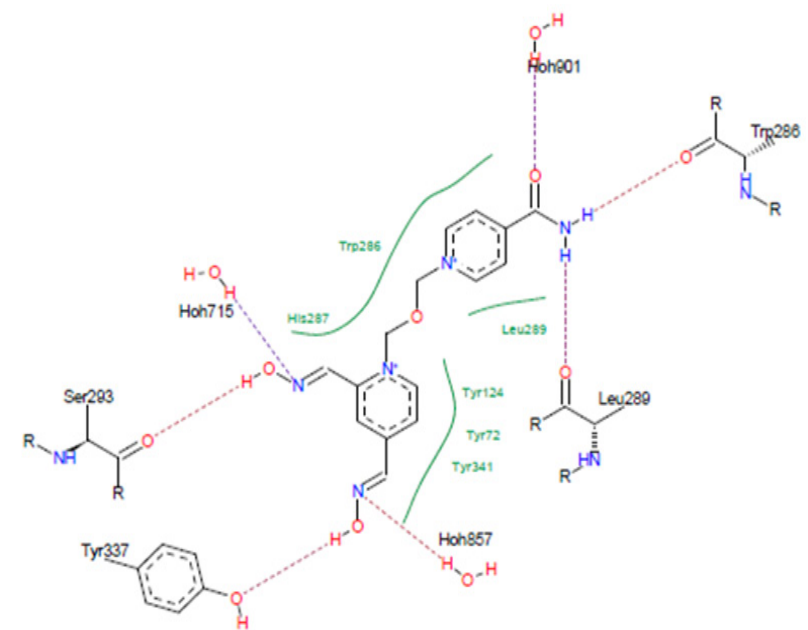

Figure 2. The docked poses of compound HLO within the binding pocket of AChE showing their different interactions (hydrogen bonds are shown in dotted lines and hydrophobic interactions are shown in green lines)

(a)

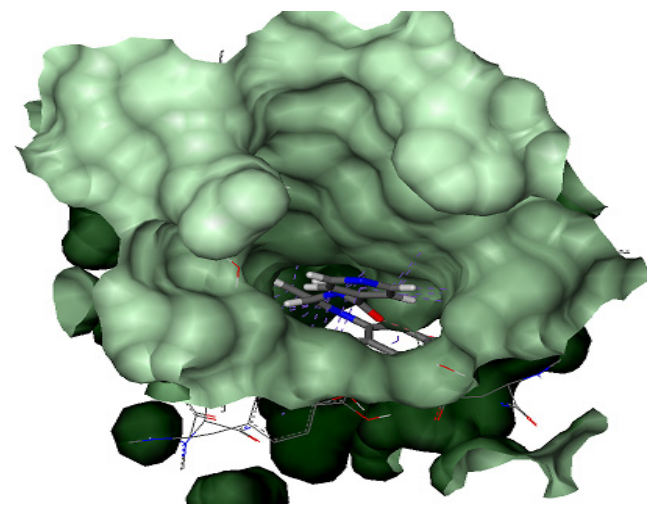

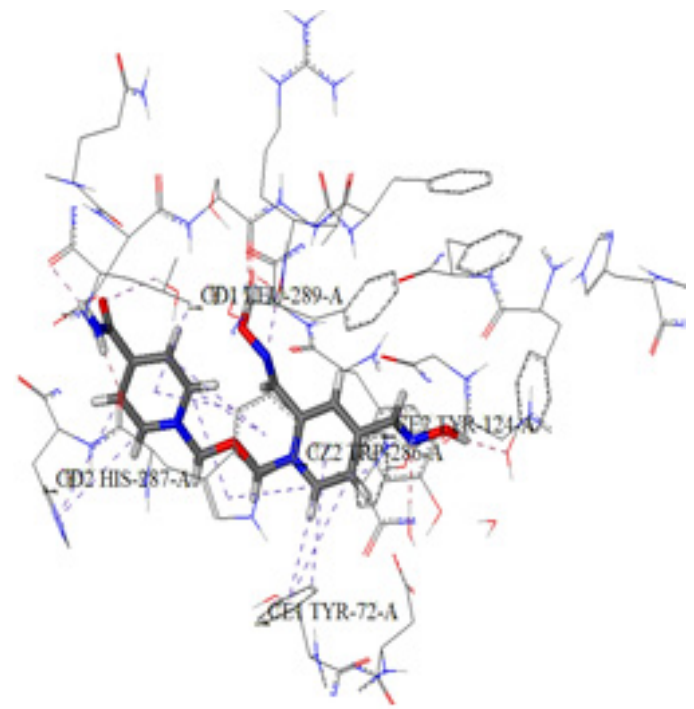

Figure 3. Representation of hydrophobic interactions formed by the inhibitor HLO

(b)

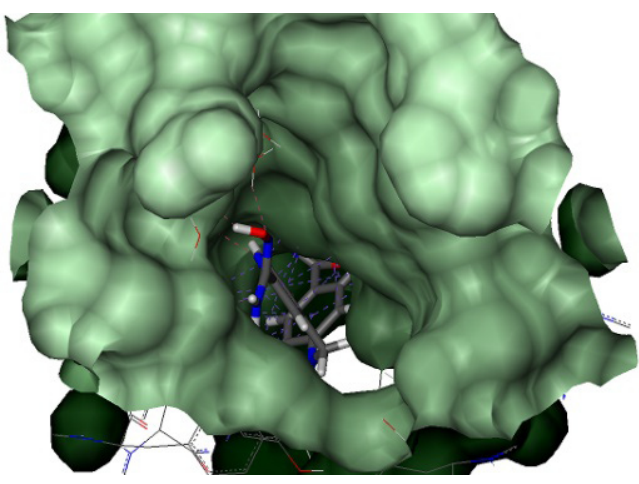

Figure 4. Illustration of the positioning of compounds ZINC00123616 (a) and ZINC78954474 (b) in the binding site of AchE

Three hydrophobic pockets stabilize the compound ZINC78954474 in the active site of AchE. Moreover, hydrophobic interactions are carried out with residues Gly121, Gly122, Tyr124, Trp286, Phe297, Tyr337, Phe338, Tyr341 and His447. In addition, the compound ZINC78954474 forms three hydrogen bonds with the binding site of AChE.

Previous studies explained the importance of the catalytic triad residues Ser203, Glu334 and His447 in the AChE active site [13] and also described the involvement of Tyr72, Tyr124 and Trp286 in the ligand binding in the peripheral site [14]. That Tyr72, Tyr124, Trp286 and His447 residues lying within the active site of the AChE, showed interactions with the compound ZINC78954474 suggest the possibility of applying this product for blocking the amino acids form interacting with the $\mathrm{AChE}$ for the degradation of acetylcholine.

The docking study showed that compounds ZINC00123616 (a) and ZINC78954474 (b) fit well into the binding site of AChE (Fig. 4). The FlexX software also helped to view the number and type of interactions involved in the ZINC00123616-AchE and ZINC78954474-AchE interactions.

Interestingly, the present study revealed that the hydrogen bonding of ZINC78954474 with Ser293 and Tyr337 is similar to that of the binding orientation of HLO in the
AChE. This effect suggests that it can have a similar antiAlzheimer propriety to HLO.

In addition, compounds ZINC00123616 and ZINC78954474 showed deep links within the AChE binding pocket through their pyridine and phenyl rings. These interact by hydrophobic means with Tyr341, Tyr124, Trp286, Gly122, Gly121, Phe297, Tyr337 and Phe338.

DihydrotanshinoneI was previously reported as a potent inhibitor of human AChE. In a study with AchE target protein, this compound, however, showed selective bonding to only the peripheral site [21], where it interacts with Trp286, Tyr337, Phe338, Phe297 and Tyr341 residues. In addition, Dihydrofuran was found to form a hydrogen bridge with Tyr124 hydroxyl [18]. As ZINC00123616, also demonstrated hydrogen-bonding interactions with Tyr124 and there are no direct links between them and amino acids of the catalytic pocket except with the His447 (Fig. 5), it could be concluded that it might also behave like Dihydrotanshinone I.

Territrem B is a high affinity inhibitor of AChE, and forms numerous hydrophobic interactions with the active site residues. These include contacts between the inhibitor and the residue Trp86 and His447 in the catalytic pocket, the side chain of Tyr337 at the interface between the catalytic and peripheral pockets, and interactions involving the side 
(a)

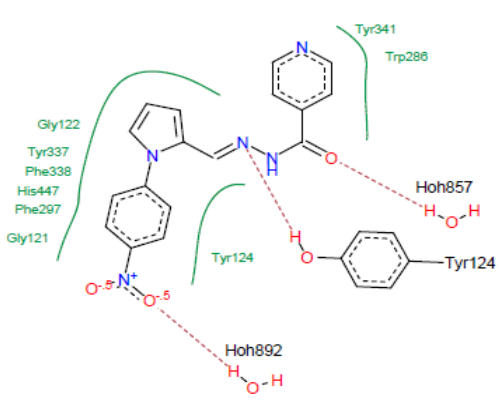

(b)

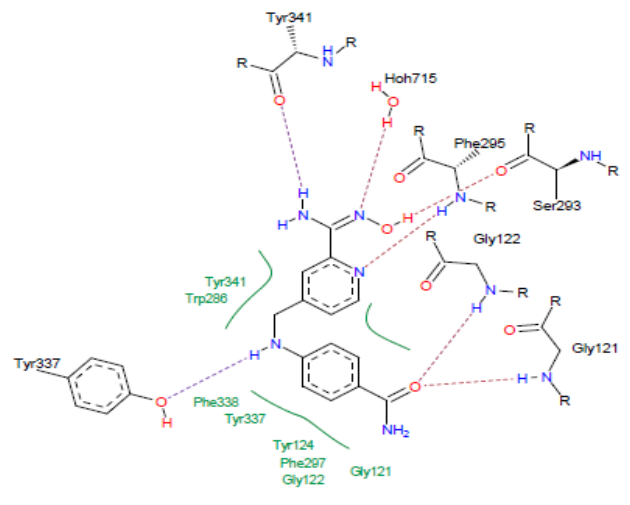

Figure 5. Interactions between ZINC00123616 (a), ZINC78954474 (b) and receptor protein residues (residue names indicated)

chains of Phe297, Tyr341and Phe338 with the inhibitor in the peripheral site [18]. These similarities in interaction of Territrem B to both the catalytic and peripheral pockets with the present studied compound, ZINC78954474, indicate that this compound is able to occupy the active site of human AChE and, hence, is involved in the inhibition of the enzyme.

\section{Drug-likeness Prediction}

In the subsequent part of our work, we verified the pharmacokinetic and toxicity proprieties of the most promising products (compounds ZINC78954474 and ZINC00123616) using Suissadme and PreADMET (Tab. 4). These properties consist of their blood-brain barrier (BBB) penetration, gastrointestinal absorption (GI), Cytochrome P450 (CYP) inhibition, Lipinski's rule of 5 catagorization, cell permeability (COCA-2) and toxicity. The same parameters of HLO were also studied for comparison.

Table 4 . The predicted pharmacokinetic and toxicity proprieties of the most promising compounds

\begin{tabular}{|c|c|c|c|}
\hline Properties & HLO & ZINC78954474 & ZINC00123616 \\
\hline BBB permeant & 0.0354779 & 0.0311664 & 0.304295 \\
\hline GI absorption & Hight & Hight & Hight \\
\hline CYP inhibitor & \begin{tabular}{|l|} 
CYP_2C9_inhibition \\
CYP_2D6_inhibition
\end{tabular} & No & $\begin{array}{l}\text { CYP2C19 inhibitor, } \\
\text { CYP2C9 inhibitor }\end{array}$ \\
\hline $\operatorname{COCA}-2^{\mathrm{a}}$ & 21.0771 & 16.9076 & 6.20373 \\
\hline Lipinski's rule of 5 & Suitable & Suitable & Suitable \\
\hline Toxicity & $\begin{array}{l}\text { hERG }{ }^{\mathrm{b}} \text { inhibition } \\
\text { (medium risk) }\end{array}$ & $\begin{array}{l}\text { hERG inhibition } \\
\text { (medium risk) }\end{array}$ & $\begin{array}{c}\mathrm{CR}^{\mathrm{c}} \\
\text { hERG inhibition } \\
\text { (medium risk) }\end{array}$ \\
\hline
\end{tabular}

As indicated in Table 4, compound ZINC00123616 is predicted to have higher $\mathrm{BBB}$ penetration than that of HLO. Moreover, the studied products have high COCA-2 cell permeability, which insures their in vivo usage. Furthermore, compound ZINC78954474 does not inhibit CYP (essential enzymes for the metabolism of numerous medicaments in the liver) contrary to HLO, which inhibits CYP2D6 and CYP2D9. With no Lipinski's rule of 5 violation, both ZINC78954474 and ZINC00123616 follow the criteria for orally available drugs. Moreover, their toxicity problems (including CR and hERG inhibition for ZINC00123616 and hERG for ZINC78954474) can be improved during their further optimization to obtain clinically useful antiAlzheimer's disease drugs.

\section{CONCLUSION}

In the present study, the molecular docking approch using FlexX was used to predict the binding energies and the interaction modes of 82 inhibitors derived from the Protein Data Bank and from a similar collection of HLO inhibitors from ZINC database for the AChE. Before performing the molecular docking, the docking protocol was validated by applying the RMSD test. This approach allowed us to suggest products ZINC00123616 and ZINC78954474 as potential new inhibitors of AChE. These proposed compounds were predicted to have good ADME/Tox properties.

\section{CONFLICT OF INTEREST}

The authors confirm that this article content has no conflicts of interest.

\section{ACKNOWLEDGEMENTS}

We are grateful to the Directorate General of Scientific Research and Technological Development, Algeria, for their support.

\section{ORCID iDs}

Merzoug Amina (1)https://orcid.org/0000-0003-2280-757X

\section{REFERNCES}

1. Atlee JL. Complications in Aanesthesia. Philadelphia: Saunders Elsevier; 2007.

2. Burns A, Iliffe S. Alzheimer's disease. BMJ. 2009;338:b158.

3. Perry EK, Tomlinson BE, Blessed G, Bergmann K, Gibson PH, Perry $\mathrm{RH}$. Correlation of cholinergic abnormalities with senile plaques and mental test scores in senile dementia. Br Med J. 1978;2(6150):1457-9.

4. Perry EK, Walker M, Grace J, Perry RH. Acetylcholine in mind: a neurotransmitter correlate of consciousness? Trends Neurosci. 1999; 22(6):273-80.

5. Draczkowski P, Tomaszuk A, Halczuk P, Strzemski M, Matosiuk D, Jozwiak K. Determination of affinity and efficacy of acetylcholinesterase inhibitors using isothermal titration calorimetry. Biochim Biophys Acta Gen Subj. 2016;1860(5):967-74.

6. Dhanjal JK, Sharma S, Grover A, Das A. Use of ligand-based pharmacophore modeling and docking approach to find novel acetylcholinesterase inhibitors for treating Alzheimer's. Biomed Pharmacother. 2015;71:146-52. 
7. Chigurupati S, Selvaraj M, Mani V, Selvarajan KK, Mohammad JI, Kaveti B, et al. Identification of novel acetylcholinesterase inhibitors: indolopyrazoline derivatives and molecular docking studies. Bioorg Chem. 2016;67:9-17.

8. Lane RM, Kivipelto M, Greig NH. Acetylcholinesterase and its inhibition in Alzheimer disease. Clin Neuropharmacol. 2004;27:141-9.

9. Galisteo M, Rissel M, Sergent O, Chevanne M, Cillard J, Guillouzo A, et al. Hepatotoxicity of tacrine: occurrence of membrane fluidity alterations without involvement of lipid peroxidation. J Pharmacol Exp Ther. 2000;294:160-7.

10. Birks J. Cholinesterase inhibitors for Alzheimer's disease. Cochrane Db Syst Rev. 2006;1:CD005593.

11. Alldredge KB. Applied therapeutics: The clinical use of drugs. $10^{\text {th }}$ ed. Baltimore: Wolters Kluwer Health/Lippincott Williams \& Wilkins; 2013.

12. Chitranshi N, Gupta S, Tripathi PK, Seth PK. New molecular scaffolds for the design of Alzheimer's acetylcholinesterase inhibitors identified using ligand- and receptor-based virtual screening. Med Chem Res. 2013;2:2328-45.

13. Quinn DM. Acetylcholinesterase: Enzyme structure, reaction dynamics, and virtual transition states. Chem Rev. 1987;87:955-75.

14. Szegletes T, Mallender WD, Thomas PJ, Rosenberry TL. Substrate binding to the peripheral site of acetylcholinesterase initiates enzymatic catalysis. Substrate inhibition arises as a secondary effect. Biochemistry. 1999;38:122-33.
15. Bourne Y, Taylor P, Radic Z, Marchot P. Structural insights into ligand interactions at the acetylcholinesterase peripheral anionic site, $E M B O$ J. 2003;22:1-12.

16. Dvir H, Silman I, Harel M, Rosenberry TL, Sussman JL. Acetylcholinesterase: From 3D structure to function. Chem Biol Interact. 2010;187:10-22.

17. Stahura FL, Bajorath J. Virtual screening methods that complement HTS. Comb Chem High Throughput Screen. 2004 ;7:259-69.

18. Cheung J, Gary EN, Shiomi K, Rosenberry TL. Structures of human acetylcholinesterase bound to dihydrotanshinone I and territrem B show peripheral site flexibility. ACS Med Chem Lett. 2013;11:1091-6.

19. Lipinski CA, Lombardo F, Dominy BW, Feeney PJ. Experimental and computational approaches to estimate solubility and permeability in drug discovery and development settings. Adv Drug Deliv Rev. 2001:46:3-26.

20. Grosdidier A. Conception d'un logiciel de docking et applications dans la recherche de nouvelles molécules actives. $\mathrm{PhD}$ Thesis : Joseph Fourier University, France; 2007.

21. Beri V, Wildman SA, Shiomi K, Al-Rashid Z, Cheung J, Rosenberry TL. The natural product dihydrotanshinone I provides a prototype for uncharged inhibitors that bind specifically to the acetylcholinesterase peripheral site with nanomolar affinity. Biochem. 2013; 52: 7486-99. 